\title{
Annual Wellness Visits in the Era of Value-Based Care: National Trends in Use, 2011-2018
}

J Gen Intern Med 36(9):2894-6

DOI: $10.1007 /$ s11606-020-06032-5

(C) Society of General Internal Medicine 2020

\section{INTRODUCTION}

In 2011, Medicare introduced the Medicare Annual Wellness Visit (AWV), which encourages clinicians to develop prevention plans, perform health risk assessments, and refer Medicare beneficiaries to preventive services. ${ }^{1}$ While there were early increases in AWV utilization between 2011 and 2014, ${ }^{2}$ little is known about longer-term trends during the era of the Medicare Access and CHIP Reauthorization Act of 2015 (MACRA) — an important gap given the increase in valuebased payment models under MACRA and potential to use AWVs to increase revenue ${ }^{3}$ and lower total healthcare spending in these models. ${ }^{4}$

\section{METHODS}

We described trends in AWV use via 2011-2018 Medicare data representing $100 \%$ of the services delivered by clinicians nationwide. We identified AWVs via Current Procedural Terminology (CPT) codes G0438 (initial AWV) and G0439 (subsequent AWV) and excluded CPT G0468, a code used at federally qualified health centers to reflect both AWVs and initial preventive physical examinations.

Given the salience of AWVs to clinicians in cognitive specialties, we described use over time among primary care (family practice, internal medicine, general practice and geriatrics), neurology, infectious disease, cardiology, nephrology, oncology, pulmonology, endocrinology, and psychiatry physicians. Given our a priori hypothesis that AWVs might be especially relevant in value-based reforms, we compared initial AWV use before (20112014) and after (2015-2018) the implementation of MACRA in 2015 by using a Kruskal-Wallis test considered significant at an alpha level of 0.05 . Institutional review board approval was not obtained given the publicly available, de-identified nature of our study data.

Received June 10, 2020

Revised June 10, 2020

Accepted July 1, 2020

Published online July 22, 2020

\section{RESULTS}

AWV use increased from 2.58 million visits in 2011 to 9.56 million visits in 2018 (Fig. 1, panel a). From 2012 onward, subsequent AWVs were more frequently billed than initial AWVs (Fig. 1, panel b). Furthermore, the number of initial AWVs performed did not differ between the pre-MACRA and post-MACRA periods (average of $1,454,443$ vs $1,182,442$ visits, respectively, $P=0.15$ ).

Primary care physicians performed the majority of AWVs throughout this time period; among physicians in other specialties, cardiologists delivered the most AWVs (Table 1). Beginning in 2012, initial AWVs were more likely to be denied than subsequent AWVs (Fig. 1, panel c). The percentage of total AWV claims that were denied declined over time, from a peak of $14.0 \%$ in 2012 to $10.5 \%$ in 2018 .

\section{DISCUSSION}

To our knowledge, this is the first analysis to describe long-term AWV use in the post-MACRA era. It demonstrates that although AWV use is increasing overall, the number of initial AWVs performed over time did not. These findings are contextualized by the relative increase in subsequent AWVs over initial AWVs and the increased likelihood of having an initial AWV denied.

The likelihood of denials for initial AWVs may disincentivize clinicians from performing them. Reasons that initial AWVs were more likely to be denied might include difficulty ascertaining whether patients have previously received an initial AWV (which can only be billed once in a patient's lifetime $)^{5}$ and confusion about the difference between initial AWVs and other types of preventive visits. Alternatively, the greater increase in subsequent AWVs may reflect longitudinal delivery of AWVs by some physicians and limited uptake among others.

Another notable result is that while primary care physicians performed the majority of AWVs, visits were also provided by subspecialists - a relevant finding given that value-based payment models encourage coordination between primary care and subspecialty clinicians without stipulating which specialties should provide preventive services. Further work is needed to evaluate the role and impact of subspecialist provision of AWVs, as well as the dynamics of initial and subsequent AWV use and denials. Broader AWV adoption may require reducing claim denials and addressing other barriers such as compliance burden (e.g., need to meet multiple requirements in a visit), difficulty 


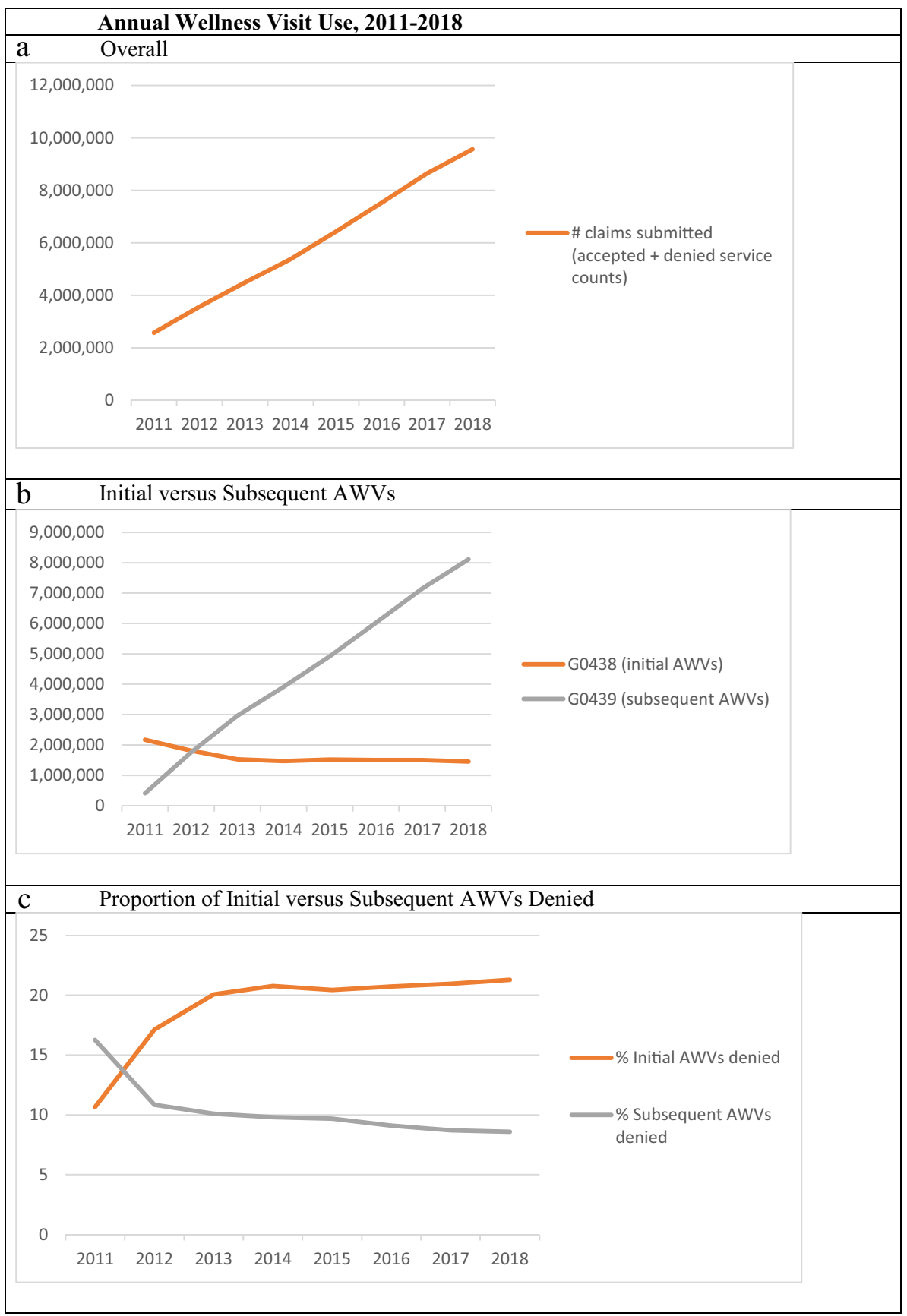

Figure 1 Annual wellness visit use, 2011-2018. Panel a Overall. Panel b Initial versus Subsequent AWVs. Panel c Proportion of initial versus subsequent AWVs denied.

Table 1 Annual Wellness Visit Use, by Specialty

\begin{tabular}{llllll}
\hline \hline Year & $\begin{array}{l}\text { Primary care*, } \boldsymbol{n} \text { (\% } \\
\text { of total) }\end{array}$ & $\begin{array}{l}\text { Cardiology, } \boldsymbol{n} \text { (\% of } \\
\text { total) }\end{array}$ & $\begin{array}{l}\text { Infectious disease, } \boldsymbol{n} \\
\text { (\% of total) }\end{array}$ & $\begin{array}{l}\text { Nephrology, } \boldsymbol{n}(\% \text { of } \\
\text { total) }\end{array}$ & $\begin{array}{l}\text { Endocrinology, } \boldsymbol{n}(\% \text { of } \\
\text { total) }\end{array}$ \\
\hline 2011 & $2,319,776(90.07)$ & $13,425(0.52)$ & $5606(0.22)$ & $5603(0.22)$ & $4528(0.18)$ \\
2018 & $8,226,553(86.02)$ & $34,645(0.55)$ & $7262(0.16)$ & $16,134(0.22)$ & $8711(0.16)$ \\
\hline
\end{tabular}

*Includes family practice, internal medicine, general practice, and geriatrics. **Includes cardiology, endocrinology, infectious disease, nephrology, neurology, oncology, preventive medicine, psychiatry, pulmonology, and others 
determining whether a patient has had an initial AWV, and limited financial incentives (e.g., subsequent AWVs and return office visits [CPT 99214] can be billed at the same rate). ${ }^{6}$

Limitations of our analysis include a descriptive design, lack of clinician- or patient-level data, and inability to assess reasons for denials. Nonetheless, our results provide unique insights into AWV use over time and highlight key areas for future research and policy.

Katherine Majzoub Morgan, $M D, M P P^{1}$

Leah M. Marcotte, $M D^{1}$

Lingmei Zhou, MS ${ }^{1}$

Joshua M. Liao, MD, MSc ${ }^{1}$

${ }^{1}$ Department of Internal Medicine, University of Washington,

Seattle, WA, USA

Corresponding Author: Katherine Majzoub Morgan, MD, MPP; Department of Internal Medicine, University of Washington Seattle, WA, USA (e-mail: kaperez@uw.edu).

\section{Compliance with Ethical Standards:}

Conflict of Interest: Dr. Morgan reports receiving income from The Everett Clinic during the conduct of the study; Dr. Marcotte reports being employed as a physician from 9/2015 to 6/2018 by Iora Health, a for-profit organization; Dr. Zhou reports no conflicts of interest; Dr. Liao reports personal fees from Kaiser Permanente Washington Research Institute and another from Wolters Kluwer, outside the submitted work.

\section{REFERENCES}

1. Medicare Learning Network. Annual Wellness Visit. Centers for Medicare and Medicaid Services. Published 2019. Available at: https://www.cms. gov/Outreach-and-Education/Medicare-Learning-Network-MLN/ MLNProducts/Downloads/AWV_Chart_ICN905706.pdf Accessed 17 Nov 2019.

2. Ganguli I, Souza J, McWilliams JM, Mehrotra A. Trends in Use of the US Medicare Annual Wellness Visit, 2011-2014. JAMA. 2017;317(21):22332235.

3. Porter S. AWV Uncorks New Revenue Stream, Improves Health Care Quality. In: AAFP; 2017. Available at: https://www.aafp.org/news/ macra-ready/20170224awv.html. Accessed 28 April 2020.

4. Beckman AL, Becerra AZ, Marcus A, et al. Medicare Annual Wellness Visit association with healthcare quality and costs. Am J Manag Care. 2019;25(3):e76-e82.

5. Chapter 18: Preventive and Screening Services. In: Medicare Claims Processing Manual. Centers for Medicare and Medicaid Services; 2019.

6. Physician Fee Schedule Search. Centers for Medicare and Medicaid Services. Published 2019. Available at: https://www.cms.gov/apps/physician-fee-schedule. Accessed Nov 18, 2019.

Publisher's Note: Springer Nature remains neutral with regard to jurisdictional claims in published maps and institutional affiliations. 